\title{
Channels Coordination Game Model Based on Result Fairness Preference and Reciprocal Fairness Preference: A Behavior Game Forecasting and Analysis Method
}

\author{
Chuan Ding, ${ }^{1}$ Kaihong Wang, ${ }^{1}$ and Xiaoying Huang ${ }^{2}$ \\ ${ }^{1}$ School of Economic Mathematics, Southwestern University of Finance and Economics, Chengdu 610074, China \\ ${ }^{2}$ School of Business Administration, Southwestern University of Finance and Economics, Chengdu 610074, China \\ Correspondence should be addressed to Chuan Ding; dingchuan@swufe.edu.cn
}

Received 7 May 2014; Revised 3 August 2014; Accepted 24 August 2014; Published 13 October 2014

Academic Editor: Li Guo

Copyright ( $\odot 2014$ Chuan Ding et al. This is an open access article distributed under the Creative Commons Attribution License, which permits unrestricted use, distribution, and reproduction in any medium, provided the original work is properly cited.

In a distribution channel, channel members are not always self-interested, but altruistic in some conditions. Based on this assumption, this paper adopts a behavior game method to analyze and forecast channel members' decision behavior based on result fairness preference and reciprocal fairness preference by embedding a fair preference theory in channel research of coordination. The behavior game forecasts that a channel can achieve coordination if channel members consider behavior elements. Using the behavior game theory model we established, we can prove that if retailers only consider the result fairness preference and they are not jealous of manufacturers' benefit, manufacturers will be more friendly to retailers. In such case, the total utility of the channel is higher compared with that of self-interest channel, and the utility of channel members is Pareto improved. If both manufactures and retailers consider reciprocal fairness preference, the manufacturers will give a lower wholesale price to the retailers. In return, the retailers will also reduce retail prices. Therefore, the total utility of the channels will not be less than the total utility of the channel coordination, as long as the reciprocity wholesale prices meet certain conditions.

\section{Introduction}

Sichuan Langjiu Group Co. Ltd. claimed in a statement on September 2, 2013, that they already terminated their coordination with Sichuan 1919 Chain Co. Ltd., and they would not provide warranty and after-sales service to the wine sold by physical store or online stores of Sichuan 1919 Chain Co. Ltd. However, Sichuan 1919 Chain Co. Ltd. held a press conference on September 3, 2013, emphasizing that the producer should be responsible for its products. See http://money .scol.com.cn/html/2013/09/017021-1150325.shtml. There was a conflict between GREE and GOME in 2004. The lack of coordination led to damage in profits of both sides. GOME and GREE also found that conflicts in the past few years resulted in a detriment to their profits. Therefore, they shook hands in 2007 [1]. Aamoco's franchisees eagerly required to decrease the rate of royalty from $9 \%$ to $5 \%$ and, in the meantime, expand their business area. By doing so, Aamoco hoped to increase the rate of royalty. An intense channel conflict happened due to the disparity of the two goals [2]. Finally, the conflict led to decreasing profits on both sides.

The above three typical cases indicated that no-coordination price mechanism led to manufacturer's and retailers' no-coordination, because the channel was in conflict. The conflict of distribution channel for both sides resulted in great loss.

Therefore, this study aims to solve such a problem: how channel coordination could be realized. Under perfect rationality, there were some coordination mechanisms, such as quantity discount, two-part tariff mechanism, and three-part tariff mechanism. However, in practice, channel members have bounded rationality. Therefore, it is necessary to design a channel coordination mechanism under bounded rationality. Behavior game is a common analysis and forecasting method, which can forecast the decision-making behavior of channel members by analyzing the behavior elements. Behavior elements include fairness preference, and bounded rationality also includes fairness preference. Empirical research and 
experimental research have shown that channel members have fairness preference. Fairness preference includes result fairness preference and reciprocal fairness preference.

The first objective of this paper is to design a channel coordination mechanism and forecast the decision behavior of channel members with result fairness preference. Research shows that if retailers only consider result fairness preference, and they are not jealous of manufacturers' benefit, manufacturers will be more friendly to retailers. In such case, the total utility of the channel should be higher compared with that of self-interest channel, and the utility of channel members is Pareto improved.

The second objective is to design a channel coordination mechanism and forecast the decision behavior of channel members with reciprocal fairness preference. To our best knowledge, no scholars forecast channel coordination using reciprocal fairness preference, there are only some channel coordination literatures of result fairness preference; see Section 2. In this paper, we propose a new reciprocal in the channel. If manufacturers give retailers lower wholesale prices, that is, manufacturers are friendly to retailers, the retailers will set lower retail price and higher marketing efforts (about marketing efforts, the author has discussed them in another paper) to repay the manufacturers; i.e., retailers are friendly to manufacturer, when the demand function is a decreasing function of the retail price. Therefore, the sales volume will increase and the profits of manufacturers and retailers will be further improved.

The third objective is to further forecast and analyze the decision behavior of channel members in the aforementioned two types of fair models. To our best knowledge, this problem has not been well studied. Therefore, the core questions are as follows. Which type the manufacturers like? What conditions should it meet? We also make some contribution to solve those two questions in this study.

\section{Literature Review}

Currently academics have focused on designing some mechanisms or contracts to achieve channel coordination of the manufacturers and retailers such as quantity discount mechanism [3], two-part tariff mechanism [4-6], three-part tariff mechanism [4], and other some complexly contract mechanisms [7-10]. Although these mechanisms could theoretically achieve the channel coordination, it was difficult to apply these mechanisms to practice. Holmstrom and Milgrom [11] believed that, in reality, a simple contract was optimal. The contract mechanisms had a basic assumption that manufacturers and retailers were perfect rationality; that is, manufacturers and retailers were assumed to maximize their own utility. However, ultimatum game, dictator game, gift exchange game, and trust game forecasted that not all channel members maximized their utility. Several prior researches [12-14] suggested that sometimes makers were altruistic. Besides, makers also considered whether they would be treated fairly by comparing their income. Arrow [15], Samuelson [16], and Sen [17] pointed out that, in reality, people were limitedly egoistic and often considered the interests of others and were also concerned about whether the allocation of material interests was fair or not. Kahneman et al. [18] argued that as individuals, business relationships, including the channel relationship when confronted with some important events, also cared about fairness, because fairness played an important role in establishing and maintaining channel relationships. That is the fairness preference in the behavioral economics and also is in fact a behavior game method. A behavior game method is a new forecasting method and decision method, in the channel management and supply chain management field, and many researchers use it to forecast the behavior of channel members or the supply chain members; Xing et al. [19], Wang and Hou [20], Du et al. [21], and $\mathrm{Ma}$ [22] forecasted and analyzed the behavior of supply chain members. So, applying the fairness preference theory into channel studies could reduce the double marginalization problem and helped the channel to realize coordination [23$25]$, which other researches related to; see $[26,27]$. Therefore, the channel coordination based on fairness preference theory became the key direction of the research.

Current studies are mainly focused on constructing the utility function of manufacturers and retailers based on the fairness preference theory. In such studies, utility function was not only to reflect the substance income, that is, without considering the fairness preference, but also to reflect the fairness preference of manufacturers and retailers; that is, utility function reflected both their income and others' income. Fairness preference of manufacturers and retailers mainly reflected two aspects.

The first one was that manufacturers and retailers were concerned whether the final result was fair or not. In practice, the manufacturers or retailers cared about material interests results, of course, not only the material interests. Fehr and Schmidt [14] proposed simple linear utility function, and we thought this fairness preference was based on the result. Fairness preference based on result assumed that the manufacturers or retailers were faced with a tradeoff between their own interests and the retailers or manufacturers' benefits; that is, the manufacturers or retailers made a maximization of individual utility between the material interests and the allocation result. Cui et al. [23] assumed that the demand function was a linear function model based on the result fairness preference which was studied, and the research showed that channel coordination was achieved by simple wholesale price contract. Caliskan-Demirag et al. [28] assumed that the demand function was nonlinear exponential and channel coordination problem was studied based on the result fairness preference. Ho and Zhang's [29] experiment found that if retailers had the result fairness preference, the efficiency of linear contract was higher than two-part tariff's efficiency. Ding et al. [30] constructed four models based on different range of the result fairness preference's coefficient. They thought that if there was no coordination mechanism, then the channel coordination could not be achieved in both types (the narrow self-interest and the competitive preference). And channel coordination could be achieved in the types of the avoiding unfair preference and the social welfare preferences, when a fair preference coefficient and other parameters satisfied certain conditions. Ding et al. [31] presented a quantity discount mechanism based on a result 
fairness preference for achieving channel coordination. They thought that as long as the degree of attention of retailer to manufacturer's profit and the fairness preference coefficients of retailers satisfied certain conditions, channel coordination could be achieved by setting a simple wholesale price and fixed costs.

The second one was that if the manufacturers or the retailers thought the other side was kind, they would repay the kindness. If one side believed that the other side would act viciously, malicious behavior would be their choice [32]. This was what people often said as "good for good" and "tit for tat." For example, people could sacrifice part of their income to maintain the fairness of income allocation and also sacrificed some profit to revenge for act of hostility or repay kindness $[33,34]$. In order to study the reciprocity theory, Rabin [12] constructed a game model of fairness preference payment function based on the framework of psychological game [35]. According to the fair definition of Rabin [12], if the manufacturers treated the retailers friendly, the retailers would also treat the manufacturers friendly. On the contrary, if manufacturers treated retailers unfriendly, the retailer would treat the manufacturer unfriendly, too. Then we wanted to know, did the differentiation between friendliness and unfriendliness become crucial? If the manufacturers lose their income and interest to improve the retailer's utility, it could be defined as the manufacturer treating the retailer friendly, that is, lose-win; conversely, if the manufacturers lose their utility to reduce the retailer's utility, it could be defined as the manufacturer being unfriendly to the retailer, namely, lose-no win. In fact, in the channel relationship, if channel members were willing to sacrifice their own material interests to help those who were nice to them or they were willing to sacrifice their material benefits to punish others' bad behaviors, we called it the reciprocal fairness preference. Rabin [12] applied the thought in the utility function of the mathematical model, and the key was the structure of kindness function.

The remainder of this paper is organized as follows. Section 4 provides basic models, channel coordination model, and manufacturer dominant channel with no fairness preference. In Section 5, we explain the retailer's utility function based on the result fairness preference. Section 6 is channel pricing model based on the retailer having result fairness preference. Section 7 is channel decision based on the reciprocity fairness preference. Section 8 is a comparative study of the two kinds of fair preference models. Section 9 is further forecasting and analysis of channel pricing based on reciprocity fairness preference. Section 10 is concluding remarks.

\section{Research Methods: A Behavior Game Analysis and Forecasting Method}

In this paper, we mainly adopt behavior game to analyze and forecast decision behavior of channel members. Game theory is a common forecasting method in operations research; behavioral game theory is a new branch of game theory. Camerer [36], one of the field's leading figures, uses psychological principles and hundreds of experiments to develop mathematical theories of reciprocity, limited strategizing, and learning, which help forecast what real people and companies do in strategic situations. Psychological principles include fairness preference. In the behavior game model, we use the fairness preference to forecast decision-making behavior, and the key is to construct the utility function of the decision maker. Specifically, we embed the result fairness preference and reciprocal fairness preference in the utility function of the manufacturer and the retailer. Behavior game model of channel coordination is constructed, in order to forecast the behavior of the manufacturer and the retailer.

\section{Basic Model [31]}

The manufacturer is the monopoly enterpriser in the distribution channels upstream, while the retailer is the consumer market monopoly distributor. Manufacturer's marginal price is $c$, wholesale price is $w$, the retailer has no other sales cost except wholesale price, and the retailer provides consumers with retail price $p$. The market demand function $q=a-p$ is a linear function of the retail price $p$, and $a$ is the market saturated demand and will be more than the marginal cost $c$; that is, $a>c>0$, which are the common knowledge between the manufacturer and the retailer. Manufacturer's profit function is $\pi_{M}=(w-c)(a-p)$, and the profit function of the retailer is $\pi_{R}=(p-w)(a-p)$.

As a baseline for comparison, we briefly give the distribution channel decision model without considering fairness preference. If the distribution channel is integrated, that is, the manufacturer and the retailer tend to maximize the channel profit and select the optimal retail price,

$$
p^{C *} \in \operatorname{Arg} \max _{p} \prod^{c}=\operatorname{Arg} \max _{p}(p-c)(a-p) .
$$

Equation (1)'s first-order condition is $p^{* C}=(a+c) / 2$, so the total channel profit is $\prod^{C *}=(a-c)^{2} / 4$.

If the channel members are independent, the manufacturer dominates the distribution channels, and the manufacturer and the retailer choose their wholesale price and retail price to maximize their profits.

The game sequence is as follows. The manufacturer determines the wholesale price. Then the retailer chooses whether or not to accept the contract according to wholesale price. If the retailer does not accept the contract, his profit is 0 , and the game is over. If the retailer accepts the contract, then according to the wholesale price given, the retailer determines retail price $p$ to maximize profit $\pi_{R}=(p-w)(a-$ $p)$. By using backward, we get that the first-order condition about $\pi_{R}$ is $p^{*}=(a+w) / 2, p^{*}=(a+w) / 2$ is replaced with the manufacturer's profit function, and the profit function is changed as $\pi_{M}=(w-c)(a-w) / 2$. Obviously, the optimal wholesale price is $w^{*}=(a+c) / 2$, and then we put $w^{*}=$ $(a+c) / 2$ into the retail price $p^{*}=(a+w) / 2$ to obtain subgame 
perfect Nash equilibrium (SPNE) and the optimal profit is given as follows:

$$
\begin{gathered}
p^{*}=\frac{3 a+c}{4}, \quad w^{*}=\frac{a+c}{2}, \\
\pi_{M}^{*}=\frac{(w-c)^{2}}{8}, \quad \pi_{R}^{*}=\frac{(w-c)^{2}}{16}, \\
\pi_{\text {Total }}^{*}=\frac{3(w-c)^{2}}{16} .
\end{gathered}
$$

\section{The Retailer's Utility Function Based on the Result Fairness Preference}

In the study of channel decision-making, the traditional assumption was that the manufacturer and the retailer were purely selfish preferences; that is, they only maximize individual income, while they did not pay attention to whether or not the distribution of income and behavior motivation were fair. In recent years, a series of experimental games, such as the ultimatum game, trust game, and gift exchange game, showed that maker had fairness preference in addition to selfinterest preference and was also concerned about whether the distribution of income or behavior motivation was fair. Fairness preference and self-interest preference would affect the behavior of channel members.

Fehr and Schmidt [14] proposed a simple linear utility function model, including the fairness preference of individual; this paper uses Fehr and Schmidt's model to construct the retailer's utility function based on fairness preference. For the convenience of research, this paper only studies the retailer who focuses on fairness preference. Therefore, the utility function of the retailer is

$$
U_{R}=\pi_{R}-\alpha \max \left(\eta \pi_{M}-\pi_{R}, 0\right)-\beta \max \left(\pi_{R}-\eta \pi_{M}, 0\right) .
$$

In equality (3), $\pi_{M}$ and $\pi_{R}$ are the manufacturer and the retailer's profits without considering fairness preference.

The following illustrates the significance of (3). The retailer's utility is composed of three parts: the first part is their profits, the second part $\max \left(\eta \pi_{M}-\pi_{R}, 0\right)$ is the envy disutility and $\alpha(\alpha>0)$ is envy coefficient, and the third part $\max \left(\pi_{R}-\eta \pi_{M}, 0\right)$ is the sympathy disutility and $\beta$ is the sympathy coefficient. In practice, the profits of channel members will not have equal distribution; for example, different channel members may invest differently; thus, the profit of channel members is to be different correspondingly, so we add arbitrary coefficient $\eta(0 \leq \eta \leq 1)$ to the manufacturer's profit. Further, in equality (3), $\max \left(\eta \pi_{M}-\right.$ $\left.\pi_{R}, 0\right)$ and $\max \left(\pi_{R}-\eta \pi_{M}, 0\right)$ have only one, regarding $\beta$, and the existing researches only show that the retailer pays less attention to the manufacturer's income but does not care more about their gains outstripping the manufacturer $[14,37,38]$. The experimental results from prior researches $[13,14]$ also show that, in general, the retailer's enthusiasm is very small when the manufacturer's income is less than the retailer's. This paper uses hypothesis $\beta=0[27,39]$. Therefore, (3) is reduced to

$$
U_{R}=\pi_{R}-\alpha \max \left(\eta \pi_{M}-\pi_{R}, 0\right)^{+} .
$$

In order to facilitate expression, we introduce the guidance function $\operatorname{sgn}(\cdot)$ in the two utility functions, respectively,

$$
\operatorname{sgn}(\cdot)= \begin{cases}1, & \eta \pi_{M}-\pi_{R} \geq 0 \\ 0, & \eta \pi_{M}-\pi_{R}<0\end{cases}
$$

Then the formula (4) is changed as follows:

$$
U_{R}=\pi_{R}-\alpha\left(\eta \pi_{M}-\pi_{R}\right) \operatorname{sgn}(\cdot) .
$$

\section{Channel Pricing Model Based on the Retailer Having Result Fairness Preference}

Under the manufacturer's dominance over the channel, the retailer's profits are less than the manufacturer's $\left(\pi_{M}^{*}=(w-\right.$ $\left.c)^{2} / 8, \pi_{R}^{*}=(w-c)^{2} / 16\right)$. So will the retailer think over whether to be treated fairly? In this case, we assume that the retailer has the result fairness preference thinking. Then, in this section, we study channel members' pricing under the retailer having result fairness preference, so the profit functions of the manufacturer and the retailer are as follows; in this paper, the profit is equal in value to utility, such as the manufacturer's profit; we sometimes referred to as the utility, indiscriminate treatment

$$
\begin{gathered}
\pi_{M}=(w-c)(a-p), \\
U_{R}=[1+\alpha \operatorname{sgn}(\cdot)](p-w)(a-p)-\alpha \eta(w-c)(a-p) .
\end{gathered}
$$

And the first-order conditions of (8) on the retail price are

$$
[1+\alpha \operatorname{sgn}(\cdot)](a-2 p+w)-\alpha \eta(c-w)=0 .
$$

Thus, the solution is $p^{F *}=([1+\alpha \operatorname{sgn}(\cdot)](a+w)-\alpha \eta(c-$ $w)) /(2[1+\alpha \operatorname{sgn}(\cdot)])$, and (7) can be written as follows:

$$
U_{M}=\frac{(w-c)[1+\alpha \operatorname{sgn}(\cdot)](a-w)+\alpha \eta(c-w)}{2[1+\alpha \operatorname{sgn}(\cdot)]} .
$$

The first-order conditions of (10) about $w$ are

$$
w^{F *}=\frac{[1+\alpha \operatorname{sgn}(\cdot)](a+c)+2 \alpha \eta c}{2[1+\alpha(1+\eta) \operatorname{sgn}(\cdot)]} .
$$

The wholesale price is replaced with the retail price, and the retail price is $p^{F *}=(3 a+c) / 4$. 
Proposition 1. If the retailer has the result fairness preference, SPNE, the manufacturer's profit, the retailer's profit, and channel total profit are

$$
\begin{aligned}
& w^{F *}= \begin{cases}\frac{a+c}{2}, & \operatorname{sgn}(\cdot)=0, \\
\frac{(1+\alpha)(a+c)+2 \alpha \eta c}{2(1+\alpha+\alpha \eta)}, & \operatorname{sgn}(\cdot)=1,\end{cases} \\
& p^{F *}=\frac{3 a+c}{4}, \\
& U_{M}^{F *}= \begin{cases}\frac{(a-c)^{2}}{8}, & \operatorname{sgn}(\cdot)=0, \\
\frac{(1+\alpha)(a-c)^{2}}{8(1+\alpha+\alpha \eta)}, & \operatorname{sgn}(\cdot)=1,\end{cases} \\
& U_{R}^{F *}= \begin{cases}\frac{(a-c)^{2}}{16}, & \operatorname{sgn}(\cdot)=0, \\
\frac{(1+\alpha)(a-c)^{2}}{16}, & \operatorname{sgn}(\cdot)=1,\end{cases} \\
& U_{\text {Total }}^{F *}=\left\{\begin{array}{l}
\frac{3(a-c)^{2}}{16}, \\
\operatorname{sgn}(\cdot)=0, \\
\frac{(a-c)^{2}}{4}+\frac{(a-c)^{2}\left(\alpha^{2}+\alpha^{2} \eta-1-3 \alpha \eta\right)}{16(1+\alpha+\alpha \eta)}, \\
\operatorname{sgn}(\cdot)=1 .
\end{array}\right.
\end{aligned}
$$

\section{Channel Pricing Model Based on Reciprocity Fairness Preference}

Since Section 5 focuses on the result fairness preference, this section will continue to study the second kind of fairness preference model in which the channel members' intention must be equal and fair. Under this circumstance, we assume that both sides of the channel have Rabin's "reciprocity" behavior [12].

The natural idea for the manufacturer is how to design his or her wholesale price in order to stimulate the retailer to actively reduce the retail price of the products to improve the product sales. In this model, a question is how to characterize the reciprocity between the manufacturer and the retailer in the model. Rabin [12] had proposed a mutual method to solve this problem.

According to actual channel, we decide to apply another method to describe different situations.

Firstly, we assume that the manufacturer knows that the retailer is bounded rationality and shows a "reciprocity"; when the manufacturer sacrifices their own interests to give the retailer more benefits, the retailer is willing to return his own interests to the manufacturer. Specifically, the manufacturer can reduce the wholesale price for the retailer. In this way, the manufacturer decides to give up a portion of the profits to the retailer. In return, the retailer will reduce the retail price appropriately.
Based on this theory, we can assume that the wholesale's price without considering the reciprocity is $w^{*}$; see Section 3. If the manufacturer reduces the part on the wholesale price $w^{*}$, the wholesale price after decreasing is $w^{*}-w^{0}$. Supposing that the retailer's "reciprocity" reaction is to reduce the retail price, so the retailer chooses the optimal retail price under the manufacturer's wholesale price $w^{*}-w^{0}$, so $\pi_{R}=(p-$ $\left.w^{*}+w^{0}\right)(a-p)$, on account of $w^{*}=(a+c) / 2$, and then $\pi_{R}=\left(p-(a+c) / 2+w^{0}\right)(a-p)$. The first-order condition for the retail price is $\tilde{p}^{F *}=(3 a+c) / 4-\left(w^{0} / 2\right)$. We can see that the retail price is reduced, so the profit functions of the manufacturer and the retailer are $\tilde{\pi}_{M}^{F *}=(1 / 2)\left[(a-c)^{2} / 4-\right.$ $\left.\left(w^{0}\right)^{2}\right], \tilde{\pi}_{R}^{F *}=\left((a-c) / 4+w^{0} / 2\right)^{2}$. As a result, there comes out a Proposition 2.

Proposition 2. If channel members have the reciprocal fairness preference, the subgame perfect Nash equilibrium (SPNE) and the optimal profits are

$$
\begin{gathered}
w^{*}=\frac{a+c}{2}, \quad \tilde{p}^{F *}=\frac{3 a+c}{4}-\frac{w^{0}}{2}, \\
\tilde{\pi}_{M}^{F *}=\frac{1}{2}\left[\frac{(a-c)^{2}}{4}-\left(w^{0}\right)^{2}\right], \\
\tilde{\pi}_{R}^{F *}=\left(\frac{a-c}{4}+\frac{w^{0}}{2}\right)^{2}, \\
\tilde{\pi}_{\text {Total }}^{F *}=\frac{(a-c)^{2}}{4}+\frac{\left[2 w^{0}-(a-c)\right]^{2}}{16} .
\end{gathered}
$$

\section{Static Comparative Analysis of Channel Pricing Decision}

Under the four models we mentioned before, simple model of channel coordination, manufacturer leading channel pricing model, channel pricing model based on result fairness preference, and channel pricing model based on reciprocity fairness preference, we should consider the following.

(1) How to change wholesale price that the manufacturer gives the retailer's?

(2) How to decide retail price?

(3) How to change the manufacturer's profit (utility)?

(4) How to adjust the retailer's profit (utility)?

(5) Compared with the general model, whether is it a Pareto improvement after introducing fairness preference?

(6) How does the fairness preference coefficient (or mutual price) affect the profit (utility) of the manufacturer, the retailer's profit (utility), and the total channel profit (utility)?

The following conclusions are to answer the 6 questions.

Conclusion 1. In three cases (the manufacturer leading channel, channel pricing model based on result fairness preference, and channel pricing model based on reciprocity fairness 
preference), the wholesale price that the manufacturer gives the retailer satisfies the following relations:

(1) when $(a-c) \alpha \eta / 2(1+\alpha+\alpha \eta) \leq w^{0}$, it holds that $\widetilde{w}^{F *} \leq$ $w^{F *} \leq w^{*}$

(2) when $(a-c) \alpha \eta / 2(1+\alpha+\alpha \eta) \geq w^{0}$, it holds that $w^{F *} \leq$ $\widetilde{w}^{F *} \leq w^{*}$.

Proof. By Propositions 1 and 2 and Section 3,

$$
\begin{gathered}
p^{F *}=\frac{3 a+c}{4}, \quad w^{*}=\frac{a+c}{2}, \\
\widehat{w}^{*}=\frac{a+c-w^{0}}{2}, \quad p^{F *}=\frac{3 a+c}{4}, \\
w^{F *}=\left\{\begin{array}{lr}
\frac{a+c}{2}, & \operatorname{sgn}(\cdot)=0, \\
\frac{(1+\alpha)(a+c)+2 \alpha \eta c}{2(1+\alpha+\alpha \eta)}, & \operatorname{sgn}(\cdot)=1 .
\end{array}\right.
\end{gathered}
$$

Obviously $\widetilde{w}^{F *} \leq w^{0}$, we need to compare the relationship between $w^{F *}$ and $w^{*}$. Because $\operatorname{sgn}(\cdot)$ 's value either is 0 or 1 , when $\operatorname{sgn}(\cdot)=0$, it holds that $w^{*}=(a+c) / 2$, when $\operatorname{sgn}(\cdot)=1$, so $w^{F *}=((1+\alpha)(a+c)+2 \alpha \eta c) /(2(1+\alpha+\alpha \eta))$; that is, $w^{F *}=$ $(a+c) / 2+\alpha \eta(c-a) / 2(1+\alpha+\alpha \eta)$, according to the previous assumption $a \geq c$, and at this time, $w^{*} \geq w^{F *}$. Next, we need to compare the relationship between $\widetilde{w}^{F *}$ and $w^{F *}$, because of $\widehat{w}^{*}=\left(a+c-w^{0}\right) / 2, w^{F *}=(a+c) / 2+\alpha \eta(c-a) / 2(1+\alpha+\alpha \eta)=$ $(a+c) / 2-\alpha \eta(a-c) / 2(1+\alpha+\alpha \eta)$, only need to compare the relationship between $w^{0}$ and $\alpha \eta(a-c) / 2(1+\alpha+\alpha \eta)$; obviously when $\alpha \eta(a-c) / 2(1+\alpha+\alpha \eta) \geq w^{0}$, it holds that $\widetilde{w}^{F *} \geq w^{F *}$, and when $\alpha \eta(a-c) / 2(1+\alpha+\alpha \eta) \leq w^{0}$, it holds that $\widetilde{w}^{F *} \leq$ $w^{F *}$. To sum up, when $\alpha \eta(a-c) / 2(1+\alpha+\alpha \eta) \leq w^{0}$, it holds that $\widetilde{w}^{F *} \leq w^{F *} \leq w^{*}$; when $\alpha \eta(a-c) / 2(1+\alpha+\alpha \eta) \geq w^{0}$, it holds that $w^{F *} \leq \widetilde{w}^{F *} \leq w^{*}$.

Conclusion 1's (1) shows that if the manufacturer's reciprocity price to the retailer $w^{0}$ is greater than a certain condition, the reciprocal fair wholesale price is the lowest. Conclusion 1's (2) shows that if the manufacturer gives reciprocity price to the retailer less than a certain condition, the result fair wholesale price is the lowest.

Conclusion 2. In four cases (channel coordination, the manufacturer dominant channel, channel pricing model based on result fairness preference, and channel pricing model based on reciprocity fairness preference), the optimal retail price satisfies the following relations:

(1) when $w^{0} \leq(a-c) / 2$, it holds that $p^{C *} \leq \tilde{p}^{F *} \leq p^{F *}=$ $p^{*}$

(2) when $w^{0} \geq(a-c) / 2$, it holds that $\tilde{p}^{F *} \leq p^{C *} \leq p^{F *}=$ $p^{*}$.

Proof. By Propositions 1 and 2 and Section 3, $p^{C *}=(a+c) / 2$, $p^{*}=(3 a+c) / 4, p^{F *}=(3 a+c) / 4$, and $\tilde{p}^{F *}=(3 a+c) / 4-w^{0} / 2$; obviously, $p^{*}=p^{F *}, p^{F *}-p^{C *}=(a+c) / 4>0$, so $p^{F *} \geq p^{C *}$. Because of $w^{0} \geq 0$, so $\widetilde{p}^{F *}=(3 a+c) / 4-w^{0} / 2 \leq(3 a+c) / 4=$
$p^{F *}$; that is, $\tilde{p}^{F *} \leq p^{F *}$. Further comparing of $p^{F *}$ and $p^{C *}$, $p^{F *}-p^{C *}=\left(a-c-2 w^{0}\right) / 4$, so, when $a-c \geq 2 w^{0}$, it holds that $p^{F *} \geq p^{C *}$. When $a-c \leq 2 w^{0}$, it holds that $p^{F *} \leq p^{C *}$. To sum up, when $a-c \geq 2 w^{0}, p^{C *} \leq \tilde{p}^{F *} \leq p^{F *}=p^{*}$. When $a-c \leq 2 w^{0}, \tilde{p}^{F *} \leq p^{C *} \leq p^{F *}=p^{*}$.

Practical significance of Conclusion 2 is very obvious, when the manufacturer dominates channels based on result fairness preference and the retailer does not return "good" to the manufacturer and not reduce his or her retail price. But when applying the channel pricing model based on reciprocity fairness preference, if the manufacturer reduces wholesale price to the retailer reciprocity, the retailer reduces retail prices to return the manufacturer. Further, the size relation of the channel integration's retail price and reciprocal retail price needs to satisfy the mutual degree of the manufacturer to the retailer; if $w^{0} \geq(a-c) / 2$, then the retail price is minimal. If the reciprocal degree is smaller $\left(w^{0} \leq(a-c) / 2\right)$, then the retail price will be greater than the coordination price.

Conclusion 3. Based on result fairness preference and reciprocity fairness preference, the manufacturer's optimal utility (profit) satisfies the following relations:

(1) $U_{M}^{F *} \geq \pi_{M}^{*}$;

(2) when $0 \leq w^{0} \leq((a-c) / 2) \sqrt{\alpha \eta /(1+\alpha+\alpha \eta)}$, it holds that $\tilde{\pi}_{M}^{F *} \geq U_{M}^{F *}$

(3) when $w^{0} \geq((a-c) / 2) \sqrt{\alpha \eta /(1+\alpha+\alpha \eta)}$, it holds that $\tilde{\pi}_{M}^{F *} \leq U_{M}^{F *}$.

Proof. Because of

$$
\begin{gathered}
U_{M}^{F *}= \begin{cases}\frac{(a-c)^{2}}{8}, & \operatorname{sgn}(\cdot)=0, \\
\frac{(a-c)^{2}(1+\alpha)}{8(1+\alpha+\alpha \eta)}, & \operatorname{sgn}(\cdot)=1,\end{cases} \\
\tilde{\pi}_{M}^{F *}=\frac{(a-c)^{2}}{8}-\frac{\left(w^{0}\right)^{2}}{2}, \\
\pi_{M}^{*}=\frac{(a-c)^{2}}{8},
\end{gathered}
$$

obviously $U_{M}^{F *} \geq \pi_{M}^{*} ; \operatorname{sgn}(\cdot)=0$ indicates that the retailer has no result fairness thinking, apparently at $\tilde{\pi}_{M}^{F *} \geq U_{M}^{F *}$. When $\operatorname{sgn}(\cdot)=1, U_{M}^{F *}=(a-c)^{2}(1+\alpha) / 8(1+\alpha+\alpha \eta)$, because of $\tilde{\pi}_{M}^{F *}-U_{M}^{F *}=\left((a-c)^{2} \alpha \eta-4\left(w^{0}\right)^{2}(1+\alpha+\alpha \eta)\right) / 8(1+\alpha+\alpha \eta)$; when $(a-c)^{2} \alpha \eta-4\left(w^{0}\right)^{2}(1+\alpha+\alpha \eta) \geq 0$, that is, when $0 \leq w^{0} \leq(a-c) / 2 \sqrt{\alpha \eta /(1+\alpha+\alpha \eta)}$, it holds that $\tilde{\pi}_{M}^{F *} \geq U_{M}^{F *}$. When $(a-c)^{2} \alpha \eta-4\left(w^{0}\right)^{2}(1+\alpha+\alpha \eta) \leq 0$, that is, when $w^{0} \geq(a-c) / 2 \sqrt{\alpha \eta /(1+\alpha+\alpha \eta)}$, it holds that $\tilde{\pi}_{M}^{F *} \leq U_{M}^{F *}$. Thus, Conclusion 3 is proved.

Conclusion 3's significance is that, under the circumstance that the manufacturer can reduce wholesale prices and gives a part of the profits to the retailer, if the decrease 
is too much $\left(w^{0} \geq(a-c) / 2 \sqrt{\alpha \eta /(1+\alpha+\alpha \eta)}\right)$, then the manufacturer would rather choose the fairness; that is to say, if the manufacturer gives the retailer too much reciprocity, it is good to himself. Only when the reciprocity level of the manufacturer satisfies the certain range $\left(0 \leq w^{0} \leq(a-\right.$ c) $/ 2 \sqrt{\alpha \eta /(1+\alpha+\alpha \eta)})$ can it find out the process benefit.

Conclusion 4. Based on result fairness preference and reciprocity fairness preference, the retailer's optimal utility (profit) satisfies the following relations:

(1) $\pi_{R}^{*} \leq U_{R}^{F *}$;

(2) when $0 \leq w^{0} \leq(a-c)(\sqrt{1+\alpha}-1) / 2$, it holds that $\tilde{\pi}_{R}^{F *} \leq U_{R}^{F *}$

(3) when $w^{0} \geq(a-c)(\sqrt{1+\alpha}-1) / 2$, it holds that $\tilde{\pi}_{R}^{F *} \geq$ $U_{M}^{F *}$.

Proof. Because of

$$
\begin{aligned}
& \pi_{R}^{*}=\frac{(a-c)^{2}}{16}, \\
& U_{R}^{F *}= \begin{cases}\frac{(a-c)^{2}}{16}, & \operatorname{sgn}(\cdot)=0, \\
\frac{(a-c)^{2}}{16}+\frac{\alpha(a-c)^{2}}{16}, & \operatorname{sgn}(\cdot)=1,\end{cases} \\
& \tilde{\pi}_{R}^{F *}=\left(\frac{a-c}{4}+\frac{w^{0}}{2}\right)^{2},
\end{aligned}
$$

obviously $\pi_{R}^{*} \leq U_{R}^{F *}$. However, $\tilde{\pi}_{R}^{F *}=(a-c)^{2} / 16+(a-c) w^{0} / 4+$ $\left(w^{0}\right)^{2} / 4$, when the retailer has fairness preference, $U_{R}^{F *}=(a-$ $c)^{2} / 16+\alpha(a-c)^{2} / 16$. So, when $(a-c) w^{0} / 4+\left(w^{0}\right)^{2} / 4 \geq \alpha(a-$ $c)^{2} / 16$, that is, when $w^{0} \geq((a-c) \sqrt{1+\alpha}-(a-c)) / 2$, it holds that $\tilde{\pi}_{R}^{F *} \geq U_{R}^{F *}$. So, when $(a-c) w^{0} / 4+\left(w^{0}\right)^{2} / 4 \leq \alpha(a-c)^{2} / 16$, that is, when $0 \leq w^{0} \leq((a-c) \sqrt{1+\alpha}-(a-c)) / 2$, it holds that $\tilde{\pi}_{R}^{F *} \leq U_{R}^{F *}$.

Part (1) of Conclusion 4 shows that retailer's gains increase by fairness preference. And the manufacturer needs to transfer a portion of the profits to the retailer, because the retailer is pursuing justice. Part (3) of Conclusion 4 shows that the retailer will pursue reciprocity fairness only when the manufacturer is willing to give the retailer reciprocity wholesale price which satisfis certain conditions.

Conclusion 5. In four cases (channel coordination, the manufacturer dominant channels, based on result fairness preference and based on reciprocity fairness preference), channel utility (profit) satisfies the following relations.

(1) Total profit channel (utility) with the retailer pursuing reciprocity fairness preference is not less than the total profit (utility) of the channel coordination (integration), that is, $\tilde{\pi}_{\text {Total }}^{F *} \geq \prod^{C *}$.

(2) When $\left(3 \eta+\sqrt{9 \eta^{2}+4(1+\eta)}\right) / 2(1+\eta) \leq \alpha \leq 1$ or $-1 \leq \alpha \leq\left(3 \eta-\sqrt{9 \eta^{2}+4(1+\eta)}\right) / 2(1+\eta)$, it holds that $U_{\text {Total }}^{F *} \geq \prod^{C *}$.
(3) When $\left(3 \eta-\sqrt{9 \eta^{2}+4(1+\eta)}\right) / 2(1+\eta) \leq \alpha \leq(3 \eta+$ $\left.\sqrt{9 \eta^{2}+4(1+\eta)}\right) / 2(1+\eta)$, it holds that $U_{\text {Total }}^{F *} \leq \prod^{C *}$.

(4) When $\left(3 \eta+\sqrt{9 \eta^{2}+4(1+\eta)}\right) / 2(1+\eta) \leq \alpha \leq 1$ or $-1 \leq \alpha \leq\left(3 \eta-\sqrt{9 \eta^{2}+4(1+\eta)}\right) / 2(1+\eta)$, it holds that $U_{\text {Total }}^{F *} \geq \tilde{\pi}_{\text {Total }}^{F *}$.

(5) When $\left(3 \eta-\sqrt{9 \eta^{2}+4(1+\eta)}\right) / 2(1+\eta) \leq \alpha \leq(3 \eta+$ $\left.\sqrt{9 \eta^{2}+4(1+\eta)}\right) / 2(1+\eta)$, it holds that $U_{\text {Total }}^{F *} \leq \tilde{\pi}_{\text {Total }}^{F *}$.

Proof. Because of

$$
\begin{gathered}
U_{\text {Total }}^{F *}=\left\{\begin{array}{l}
\frac{3(a-c)^{2}}{16}, \\
\operatorname{sgn}(\cdot)=0, \\
\frac{(a-c)^{2}}{4}+\frac{\alpha(a-c)^{2}\left(\alpha^{2}+\alpha^{2} \eta-1-3 \alpha \eta\right)}{16(1+\alpha+\alpha \eta)}, \\
\operatorname{sgn}(\cdot)=1,
\end{array}\right. \\
\prod_{\text {Total }}^{C *}=\frac{(a-c)^{2}}{4}, \quad \pi_{\text {Total }}^{*}=\frac{3(a-c)^{2}}{16}, \\
\tilde{\pi}^{F *}+\frac{\left[2 w^{0}-(a-c)\right]^{2}}{16} .
\end{gathered}
$$

(1) So the retailer pursues reciprocal fairness, and results are that total profit (utility) is not less than the channel coordination (integration) of the total profit (utility); that is, $\tilde{\pi}_{\text {Total }}^{F *} \leq \prod^{C *}$.

(2) When $(a-c)^{2}\left(\alpha^{2}+\alpha^{2} \eta-1-3 \alpha \eta\right) / 16(1+\alpha+\alpha \eta)$, that is, $\left(3 \eta+\sqrt{9 \eta^{2}+4(1+\eta)}\right) / 2(1+\eta) \leq \alpha \leq 1$ or $-1 \leq$ $\alpha \leq\left(3 \eta-\sqrt{9 \eta^{2}+4(1+\eta)}\right) / 2(1+\eta), U_{\text {Total }}^{F *} \geq \prod^{C *}$.

(3) When $\left(3 \eta-\sqrt{9 \eta^{2}+4(1+\eta)}\right) / 2(1+\eta) \leq \alpha \leq(3 \eta+$ $\left.\sqrt{9 \eta^{2}+4(1+\eta)}\right) / 2(1+\eta), U_{\text {Total }}^{F *} \leq \prod^{C *}$.

(4) $U_{\text {Total }}^{F *}$ 's second expression is not less than zero; while $\tilde{\pi}_{\text {Total }}^{F *}$ 's second is greater than zero, so $\left(3 \eta+\sqrt{9 \eta^{2}+4(1+\eta)}\right) / 2(1+\eta) \leq \alpha \leq 1$ or $-1 \leq \alpha \leq\left(3 \eta-\sqrt{9 \eta^{2}+4(1+\eta)}\right) / 2(1+\eta)$, it holds that $U_{\text {Total }}^{F *} \geq \tilde{\pi}_{\text {Total }}^{F *} ;$ when $\left(3 \eta-\sqrt{9 \eta^{2}+4(1+\eta)}\right) / 2(1+\eta) \leq$ $\alpha \leq\left(3 \eta+\sqrt{9 \eta^{2}+4(1+\eta)}\right) / 2(1+\eta)$, it holds that $U_{\text {Total }}^{F *} \leq \tilde{\pi}_{\text {Total }}^{F *}$. Conclusion 5 is proved.

Conclusion 5 shows that if the retailer only considers about reciprocity fairness preference, total channel utility gets a Pareto improvement to the general channel coordination. Combining Conclusion 3 with Conclusion 4, as long as our reciprocity wholesale price satisfies certain conditions, the manufacturer and the retailer's utility gets a Pareto 
improvement to that not considering fairness preference. When considering the result fairness preference, as long as the proportion coefficient satisfies certain conditions, the total channel utility is over the general channel utility. When considering fairness preference (whether result fairness preference or reciprocal fairness preference), channel total utility can all reach the level of channel coordination, and both sides' utility gets a Pareto improvement; thus fairness preference is important.

Next, we continue to discuss the influence of model parameters on the manufacturer, the retailer, and channel utility. From Proposition 1, we can get the following.

When considering result fairness preference, that is, $\operatorname{sgn}(\cdot)=1$, so $\partial U_{M}^{F *} / \partial \eta<0, \partial U_{M}^{F *} / \partial \alpha=-\eta(a-c)^{2} / 8(1+$ $\alpha+\alpha \eta)<0$. This shows that the higher $\eta$ is detrimental to the manufacturer, because the greater the value of $\eta$ is, the more the retailer focuses on manufacture's income. And the larger fairness preference coefficient also means more detrimental to the manufacturer, because the larger fairness preference coefficient means that the retailer pays more attention to equity issues. In order to maintain the channel coordination, the manufacturer shares a portion of the profits to the retailer.

Then, we take a look at the change of the retailer's benefits. Obviously, it is more favorable to the retailer if they pay more attention to fairness, and we can also tell that it is an increasing function for fairness preference coefficient from $U_{R}^{F *}$. This is also the power of the retailer to pursue fairness preference. Further, we consider the total channel profit; $U_{\text {Total }}^{F *}$ decreases with $\eta$ when $\alpha \in(0,1]$, because of $\partial U_{\text {Total }}^{F *} / \partial \eta=-(a-c)^{2} \alpha(1+\alpha) / 8(1+\alpha+\alpha \eta)$. This kind of logic improves the level of channel coordination. When $\alpha \in(0,1]$, $U_{\text {Total }}^{F *}$ decreases with the $\alpha$. So the retailer's jealousy reduces channel coordination levels. Thus comes Conclusion 6.

Conclusion 6. When considering result fairness preference, it is more detrimental to the manufacturer if the retailer focuses more on manufacture's income (the higher $\eta$ ). And it is also more detrimental to the manufacturer, if the retailer pays more attention to the fairness preference (larger preference coefficient). In order to maintain the channel coordination, the manufacturer will transfer a portion of the income to the retailer. Further, if the retailer is more generous, not comparing with the manufacturer, then it will improve channel coordination. Instead, the retailer's jealousy will result in reduction of channel coordination.

From Proposition 2, $\tilde{\pi}_{M}^{F *}=(1 / 2)\left[(a-c)^{2} / 4-\left(w^{0}\right)^{2}\right]$, $\tilde{\pi}_{R}^{F *}=\left((a-c) / 4+w^{0} / 2\right)^{2}, \tilde{\pi}_{\text {Total }}^{F *}=(a-c)^{2} / 4+\left[2 w^{0}-(a-c)\right]^{2} / 16$, large reciprocity wholesale price $\left(w^{0}\right)$ is detrimental to the manufacturer, but it will increase the retailer's utility (profit) and will also increase the total channel utility (profit). And here comes Conclusion 7.

Conclusion 7. Considering reciprocity fairness preference, large reciprocity wholesale price is detrimental to the manufacturer, but it will increase the retailer's utility (profit) and can also increase the total channel utility (profit).

\section{Further Forecasting and Analysis of Channel Pricing Based on Reciprocity Fairness Preference}

Conclusion 7 shows that considering that reciprocity fairness preference is detrimental to the manufacturer, if the manufacturer's utility (profit) can be guaranteed not less than the general channel utility (profit), then, can the retailer's utility (profit) and the total channel utility (profit) be improved? So the problem is actually the conditional extremism problem:

$$
\begin{gathered}
\max _{w} \pi_{M}=\max _{w}(w-c)(a-p), \\
\text { st }: p^{*} \in \max _{p} \pi_{R}=\max _{p}\left(p-w+w^{0}\right)(a-p), \\
\pi_{M}=(w-c)(a-p) \geq \frac{(a-c)^{2}}{8}=\pi_{M}^{*} .
\end{gathered}
$$

The extreme value problem (20) is the manufacturer's optimization selection, (21) is the choice of the retailer's optimal, and (22) is the basic condition for the profits of the manufacturer requirements. Proposition 3 can be obtained by the model above.

Proposition 3. The manufacturer and the retailer have the reciprocal fairness preference; if the manufacturer's utility (profit) is not less than the general channel utility (profit), then the optimal wholesale price, retail price, the manufacturer's utility (profit), the retailer's utility (profit), and the channel total utility (profit) are as follows:

$$
\begin{gathered}
\widehat{p}^{*}=\frac{3 a+c-w^{0}}{4}, \quad \widehat{w}^{*}=\frac{a+c-w^{0}}{2}, \\
\widehat{\pi}_{M}^{*}=\frac{\left(w-c+w^{0}\right)^{2}}{8}, \\
\widehat{\pi}_{R}^{*}=\frac{\left(w-c+w^{0}\right)^{2}}{16}, \quad \hat{\pi}_{\text {Total }}^{*}=\frac{3\left(w-c+w^{0}\right)^{2}}{16} .
\end{gathered}
$$

Proof. Extremism problem of deformation is

$$
\begin{gathered}
\max _{w} \pi_{M}=\max _{w}(w-c)(a-p), \\
\text { st: } p=\frac{a+w-w^{0}}{2}, \\
(w-c)(a-p) \geq \frac{(a-c)^{2}}{8} .
\end{gathered}
$$

Equation (25) is taken into (24) and (26), and extremism problem becomes the following:

$$
\begin{aligned}
& \max _{w} \pi_{M}=\max _{w}(w-c) \frac{a-w+w^{0}}{2}, \\
& (w-c) \frac{a-w+w^{0}}{2}-\frac{(a-c)^{2}}{8} \geq 0 .
\end{aligned}
$$

The K-T condition is $\left(1-\kappa^{*}\right)\left[\left(a-w^{*}+w^{0}\right) / 2-\left(w^{*}-c\right) / 2\right]=0$, $\kappa^{*}\left[\left(w^{*}-c\right)\left(\left(a-w^{*}+w^{0}\right) / 2\right)-(a-c)^{2} / 8\right]=0$, and $\kappa^{*}$ is 
a nonnegative generalized Lagrange multiplier. When $\kappa^{*}=0$, it holds that $w^{*}=\left(a+w^{0}+c\right) / 2$; when $\kappa^{*}>0$, it holds that $\left[\left(w^{*}-c\right)\left(\left(a-w^{*}+w^{0}\right) / 2\right)-(a-c)^{2} / 8\right]=0$, so

$$
\begin{aligned}
w^{*}= & \left(-\left[c+\left(a+w^{0}\right)\right]\right. \\
& \left. \pm \sqrt{\left[c+\left(a+w^{0}\right)\right]^{2}-\left[4 c\left(a+w^{0}\right)+(a-c)^{2}\right]}\right) \\
& \times(2)^{-1}
\end{aligned}
$$

to meet the requirements $\left[c+\left(a+w^{0}\right)\right]^{2} \geq\left[4 c\left(a+w^{0}\right)+(a-c)^{2}\right]$, so

$$
\begin{aligned}
& w^{*}=-\left[c+\left(a+w^{0}\right)\right] \\
&\left.+\sqrt{\left[c+\left(a+w^{0}\right)\right]^{2}-\left[4 c\left(a+w^{0}\right)+(a-c)^{2}\right]}\right) \\
& \times(2)^{-1} \\
& w^{*}=\left(-\left[c+\left(a+w^{0}\right)\right]\right. \\
&\left.\quad-\sqrt{\left[c+\left(a+w^{0}\right)\right]^{2}-\left[4 c\left(a+w^{0}\right)+(a-c)^{2}\right]}\right) \\
& \times(2)^{-1} .
\end{aligned}
$$

Then three K-T points are

$$
\begin{gathered}
w_{(1)}^{*}=\frac{a+w^{0}+c}{2}, \\
w_{(2)}^{*}=\left(-\left[c+\left(a+w^{0}\right)\right]\right. \\
\left.+\sqrt{\left[c+\left(a+w^{0}\right)\right]^{2}-\left[4 c\left(a+w^{0}\right)+(a-c)^{2}\right]}\right) \\
\times(2)^{-1}, \\
w_{(3)}^{*}=\left(-\left[c+\left(a+w^{0}\right)\right]\right. \\
\left.\quad-\sqrt{\left[c+\left(a+w^{0}\right)\right]^{2}-\left[4 c\left(a+w^{0}\right)+(a-c)^{2}\right]}\right) \\
\times(2)^{-1} .
\end{gathered}
$$

$w_{(1)}^{*}, w_{(2)}^{*}$, and $w_{(3)}^{*}$ are brought into the manufacturer's profit function

$$
\begin{gathered}
\pi_{M}\left(w_{(1)}^{*}\right)=\frac{\left(a-c+w^{0}\right)^{2}}{8}, \\
\pi_{M}\left(w_{(2)}^{*}\right)=\frac{(a-c)^{2}}{8}, \quad \pi_{M}\left(w_{(3)}^{*}\right)=\frac{(a-c)^{2}}{8} .
\end{gathered}
$$

So, $w^{*}=\left(a+c+w^{0}\right) / 2$ is the maximum value, and the maximum value is $\widehat{\pi}_{M}=\left(a-c+w^{0}\right)^{2} / 8$, and $\widehat{\pi}_{M}=(a-$ $\left.c+w^{0}\right)^{2} / 8$ is taken into $(25): \widehat{p}^{*}=\left(3 a-w^{0}+c\right) / 4$, so $\widehat{\pi}_{R}=$ $\left(a-c+w^{0}\right)^{2} / 16$, and $\hat{\pi}_{\text {Total }}=3\left(a-c+w^{0}\right)^{2} / 16$. The wholesale price $\widehat{w}^{*}=w^{*}-w^{0}=\left(a-w^{0}+c\right) / 2$, so Proposition 3 is proved.

Conclusion 8. The manufacturer and the retailer have the reciprocal fairness preference; if the manufacturer lowers the part of wholesale prices (reciprocal price $w^{0}$ ) to the retailer, the retailer will reduce the retail price as a return to the manufacturer. Thereby, it will improve the manufacturer's utility (profit), the retailer's utility (profit), and the total channel utility (profit) and will further improve the channel total utility (profit).

Proof. By Section 3 and Proposition 3, $p^{*}=(3 a+c) / 4, w^{*}=$ $(a+c) / 2, \pi_{M}^{*}=(w-c)^{2} / 8, \pi_{R}^{*}=(w-c)^{2} / 16, \pi_{\text {Total }}^{*}=3(w-$ $c)^{2} / 16, \widehat{p}^{*}=\left(3 a+c-w^{0}\right) / 4, \widehat{w}^{*}=\left(a+c-w^{0}\right) / 2, \widehat{\pi}_{M}^{*}=$ $\left(w-c+w^{0}\right)^{2} / 8, \hat{\pi}_{R}^{*}=\left(w-c+w^{0}\right)^{2} / 16, \hat{\pi}_{\text {Total }}^{*}=3\left(w-c+w^{0}\right)^{2} / 16$, obviously, we find that $p^{*} \leq \widehat{p}^{*}, w^{*} \leq \widehat{w}^{*}, \pi_{R}^{*} \leq \widehat{\pi}_{R}^{*}, \pi_{M}^{*} \leq \widehat{\pi}_{M}^{*}$, $\pi_{\text {Total }}^{*} \leq \hat{\pi}_{\text {Total }}^{*}$. Because of $\prod^{*}=(a-c)^{2} / 4, \hat{\pi}_{\text {Total }}^{*}=3(w-c+$ $\left.w^{0}\right)^{2} / 16$, so $\hat{\pi}_{\text {Total }}^{*}-\prod^{*}=\left(3\left(w^{0}\right)^{2}+6(a-c) w^{0}-(a-c)^{2}\right) / 16 \geq 0$, requiring $3\left(w^{0}\right)^{2}+6(a-c) w^{0}-(a-c)^{2} \geq 0$, that is, $w^{0} \geq(\sqrt{6}-$ $3)(a-c) / 3$, because of $w^{0} \geq 0$. So $w^{0} \geq(\sqrt{6}-3)(a-c) / 3$ must satisfy the inequality, so as long as $w^{0} \geq 0$, then $\widehat{\pi}_{\text {Total }}^{*}-\prod^{*} \geq$ 0 ; that is, $\widehat{\pi}_{\text {Total }}^{*} \geq \prod^{*} \geq 0$.

Conclusion 8 is especially meaningful. If the manufacturer is required to obtain utility (profit) not less than general utility (profit) and reduces the wholesale prices moderately to show friendship to the retailer, then the retailer will reduce retail prices as a return to the manufacturer and lowers retail price and thus increases demand. Results are increasing both utilities (profits), also increasing the total channel utility (profit). That is to say, the manufacturer can achieve channel coordination simply by setting wholesale prices, which is obviously better than complex channel coordination mechanisms. This conclusion is consistent with Xing et al. [19], but the results of Xing et al. [19] are based on result fairness preference.

Proposition 2 and Conclusion 8 illustrate that reciprocity plays an important role. The manufacturer first determines the wholesale price; then the retailer decides the retail price based on the price set by the manufacturer. Retail price is an increasing function of the manufacturer's reciprocity price $w^{0}$. That is to say, if a manufacturer is more reciprocal to the retailer, then the retailer will also give more benefits to a manufacturer. So we easily get good degree of the retailer to the manufacturer return which is $\Delta p=p^{*}-\widehat{p}^{*}=$ $w^{0} / 4$. So good faith degree $\Delta p$ of the retailer return to the manufacturer is an increasing function of reciprocal price $w^{0}$. This further explains the principle of reciprocity fairness preference.

\section{Concluding Remarks}

In the paper, channel coordination is studied based on fairness preference theory of behavioral economics. We use the new forecasting method, behavior game method. First, we 
establish a general channel decision model, which is used as a benchmark model for comparison. Then, two channel pricing models are built based on either result fairness preference or reciprocal fairness preference. The model based on reciprocal fairness preference is discussed in detail. Finally, many conclusions were predicted and tested by several behavior game models, which are listed below.

(1) When the manufacturer dominates channels and the channel is based on result fairness preference, the retailer will not reduce retail price. When channels are based on the reciprocal fairness preference and the manufacturer sets lower wholesale prices for the retailer, the retailer may reduce retail prices as a return to the manufacturer. If the reciprocal degree is high, then the retail price will be low. If the reciprocal degree is small, then the retail price will be higher than the coordination price.

(2) If the retailer only considers reciprocal fairness preference, total utility from channel is a Pareto improvement of total utility from the general channel coordination. As long as the reciprocal wholesale price is reduced to the range, the total utility of the manufacturer and the retailer is Pareto improved compared to the case when fairness preference is not introduced to the system.

(3) When the result fairness preference is introduced, the retailer will pay attention to the profits of the manufacturer and will also pay close attention to the justice problem. In order to maintain the channel coordination, the manufacturer must share some profits with the retailer. The more attention the retailer pays to justice, the more benefits the retailer gets. If the retailer is more magnanimous and does not pay too much attention to the manufacturer's profit, channel coordination will be stronger.

(4) When considering reciprocal fairness preference, greater reciprocal wholesale price is detrimental to the manufacturer but will increase the retailer's utility (profit), as well as the total channel utility (profit).

(5) When considering the reciprocal fairness preference, the manufacturer's utility (profit) is not less than utility (profit) of the general channel; if the manufacturer reduces wholesale prices to the retailer, then the retailer may also reduce the retail price, thereby improving the manufacturer's utility (profit), the retailer's utility (profit), and also the total channel utility (profit). Finally, channel total utility (profit) will become larger than the channel utility (profit).

There are also some limitations in this paper. First, this paper only studies the retailer's fairness preference; however, the manufacturer should also have fairness preference. Second, this paper only studies simple two-player game in the channel coordination problem. Third, we analyze the retailer's decision problem based on result fairness preference and reciprocal fairness preference separately, but we did not study them in a unified framework.

\section{Conflict of Interests}

The authors declare that there is no conflict of interests regarding the publication of this paper.

\section{Acknowledgments}

The authors are particularly grateful to the associate editor and reviewers for thoughtful, valuable discussions and suggestions. The authors acknowledge the financial support by Humanities and Social Science Project of Ministry of Education of China (14XJCZH001), by Soft Science Research Project of Sichuan Province (2014ZR0027), and by the Fundamental Research Funds for the Central Universities (JBK130401).

\section{References}

[1] H. X. Zhu, "Household electrical industry channel conflict and its resolution- Analysis based on the Green Electric," Jiangsu Commercial Forum, vol. 7, pp. 14-16, 2010 (Chinese).

[2] Y. T. Zhu, Channel Conflict, Management Press, 2004, (Chinese).

[3] A. P. Jeuland and S. M. Shugan, "Managing channel profits," Market Science, vol. 2, no. 3, pp. 239-272, 1983.

[4] K. S. Moorthy, "Sridhar, managing channel profits: comment," Marketing Science, vol. 6, no. 4, pp. 375-379, 1987.

[5] P. Zusman and M. Etgar, "The marketing channel as an Equilibrium Set of Contracts," Management Science, vol. 27, no. 3, pp. 284-302, 1981.

[6] T. W. McGlre and R. Staelin, "Channel efficiency, in centive compatibility, transfer pricing and market structure: an equilibrium analysis of channel relationships," in Research in Maketing, L. P. Bucklin and J. M. Carman, Eds., vol. 8, JAI Press, Greenwich, Conn, USA, 1986.

[7] H. F. Zhao, B. Lin, W. Q. Mao, and Y. Ye, "Differential game analyses of logistics service supply chain coordination by cost sharing contract," Journal of Applied Mathematics, vol. 2014, Article ID 842409, 10 pages, 2014.

[8] G. Xie, W. Yue, and S. Wang, "Quality improvement policies in a supply chain with Stackelberg games," Journal of Applied Mathematics, vol. 2014, Article ID 848593, 9 pages, 2014.

[9] G. D. Wu, Q. S. Kong, J. G. Shi, H. R. Karimi, and W. Zhang, "Information sharing and channel construction of supply chain under asymmetric demand information," Journal of Applied Mathematics, vol. 2014, Article ID 107589, 8 pages, 2014.

[10] Y. H. Chen and X. W. Wen, "Vertical cooperative advertising with substitute brands," Journal of Applied Mathematics, vol. 2013, Article ID 480401, 8 pages, 2013.

[11] B. Holmstrom and P. Milgrom, "Aggregation and linearity in the provision of intertemporal incentives," Econometrica, vol. 55, no. 2, pp. 303-328, 1987.

[12] M. Rabin, "Incorporating fairness into game theory and economics," American Economic Review, vol. 83, no. 5, pp. 1281$1302,1993$.

[13] G. Charness and M. Rabin, "Understanding social preferences with simple tests," Quarterly Journal of Economics, vol. 117, no. 3, pp. 817-869, 2002.

[14] E. Fehr and K. M. Schmidt, "A theory of fairness, competition, and cooperation," Quarterly Journal of Economics, vol. 114, no. 3, pp. 817-868, 1999 . 
[15] J. K. Arrow, "Optimal and voluntary income redistribution," in Economic Welfare and the Economics of Soviet Socialism: Essays in Honor of Abram Bergson, S. Rosefielde, Ed., Cambridge University Press, Cambridge, UK, 1981.

[16] P. A. Samuelson, "Altruism as a problem involving group versus individual selection in economics and biology," American Economic Review, vol. 83, no. 2, pp. 143-148, 1993.

[17] A. Sen, Moral Codes and Economic Success, Edward Eldar Press, Cheltenham, UK, 1995.

[18] D. Kahneman, J. L. Knetsch, and R. Thaler, "Fairness and the assumptions of economics," The Journal of Business: Part 2: The Behavioral Foundatio ns of Economic Theory, vol. 59, no. 4, pp. 285-300, 1986.

[19] W. Xing, S.-Y. Wang, Q.-H. Zhao, and G.-W. Hua, "Impact of fairness on strategies in dual-channel supply chain," System Engineering Theory and Practice, vol. 31, no. 7, pp. 1249-1256, 2011 (Chinese)

[20] H. Wang and W. H. Hou, "Design of supply chain incentive contracts with fairness preference of the suppliers considered," Logistics Technologh, vol. 17, no. 9, pp. 44-48, 2010 (Chinese).

[21] S. F. Du, C. Du, L. Liang, and T. Z. Liu, "Supply chain coordination considering fairness concerns," Journal of Management Sciences in China, vol. 13, no. 11, pp. 41-48, 2010 (Chinese).

[22] L. J. Ma, "Supply chain analysiswith fairness preference," Operations Research and Management Science, vol. 20, no. 2, pp. 37-43, 2011 (Chinese).

[23] T. H. Cui, J. S. Raju, and Z. J. Zhang, "Fairness and channel coordination," Management Science, vol. 53, no. 8, pp. 1303-1314, 2007.

[24] C. H. Loch and Y. Wu, "Social preferences and supply chain performance: an experimental study," Management Science, vol. 54, no. 11, pp. 1835-1849, 2008.

[25] Q. S. Ruan and X. H. Huang, "Theory research of western fairness preference," Foreign Economy and Management, vol. 27, no. 6 , pp. 10-16, 2005.

[26] Y.-C. Tsao, T.-H. Chen, and P.-Y. Wu, "Effects of lot-sizing integration and learning effect on managing imperfect items in a manufacturerretailer chain," Journal of Applied Mathematics, vol. 2013, Article ID 413206, 11 pages, 2013.

[27] C. Y. Chiu, Y. Lin, and M. F. Yang, "Applying fuzzy multiobjective integrated logistics model to green supply chain problems," Journal of Applied Mathematics, vol. 2014, Article ID 767095, 12 pages, 2014.

[28] O. Caliskan-Demirag, Y. Chen, and J. Li, "Channel coordination under fairness concerns and nonlinear demand," European Journal of Operational Research, vol. 207, no. 3, pp. 1321-1326, 2010.

[29] T.-H. Ho and J. Zhang, "Designing pricing contracts for boundedly rational customers: does the framing of the fixed fee matter?" Management Science, vol. 54, no. 4, pp. 686-700, 2008.

[30] C. Ding, K. H. Wang, and R. Ran, "Marketing channel coordination mechanism based on fairness preference," Journal of Management Sciences in China, vol. 16, no. 8, pp. 80-94, 2013.

[31] C. Ding, K. Wang, and S. Lai, "Channel coordination mechanism with retailers having fairness preference-an improved quantity discount mechanism," Journal of Industrial and Management Optimization, vol. 9, no. 4, pp. 967-982, 2013.

[32] J. J. Xia et al., "Altruism, trust and trust," Economic Science, vol. 27, no. 4, pp. 95-108, 2003.

[33] Y. J. Pu, "Principal-agent model based on the fair game," Modern Finance, vol. 68, no. 3, pp. 5-11, 2007.
[34] Y. J. Pu, "the principal-agent model: alternative, material utility and motivation fairness based on behavioral economics theory," Quarterly Journal of Economics, vol. 7, no. 1, pp. 298-318, 2007.

[35] J. Geanakoplos, D. Pearce, and E. Stacchetti, "Psychological games and sequential rationality," Games and Economic Behavior, vol. 1, no. 1, pp. 60-79, 1989.

[36] C. F. Camerer, Behavioral Game Theory: Experiments in Strategic Interaction, Princeton University Press, 2003.

[37] G. E. Bolton, "A comparative model of bargaining: theory and evidence," American Economic Review, vol. 81, no. 5, pp. 10961120, 1991.

[38] A. D. Bruyn and G. E. Bolton, "Estimating the influence of fairness on bargaining behavior," Management Science, vol. 54, no. 10, pp. 1774-1791, 2008.

[39] E. Valery and V. Elena, "Fairness and coordination failu res in supply chain contracts," Penn State Working Paper, 2009. 


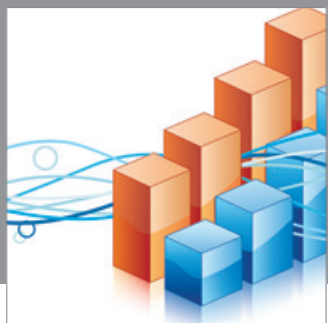

Advances in

Operations Research

mansans

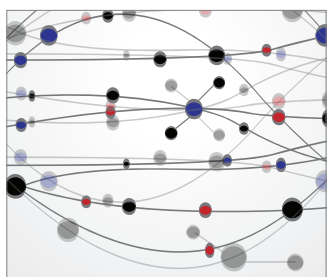

The Scientific World Journal
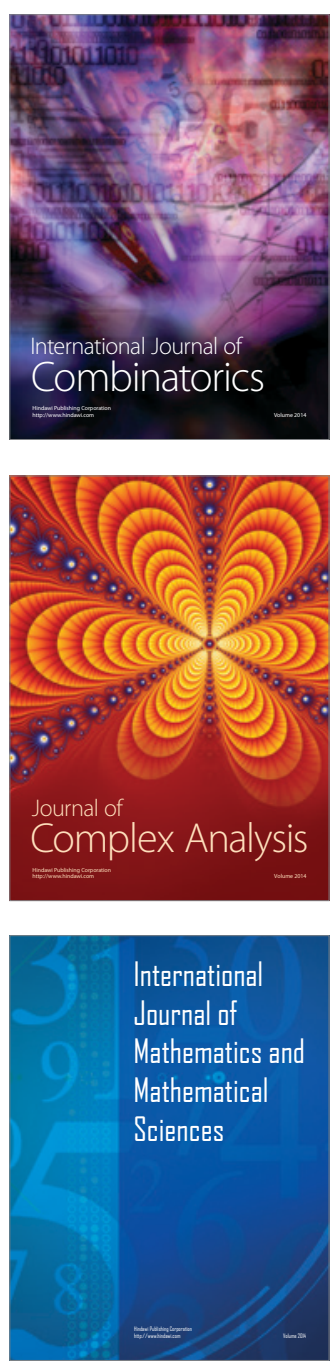
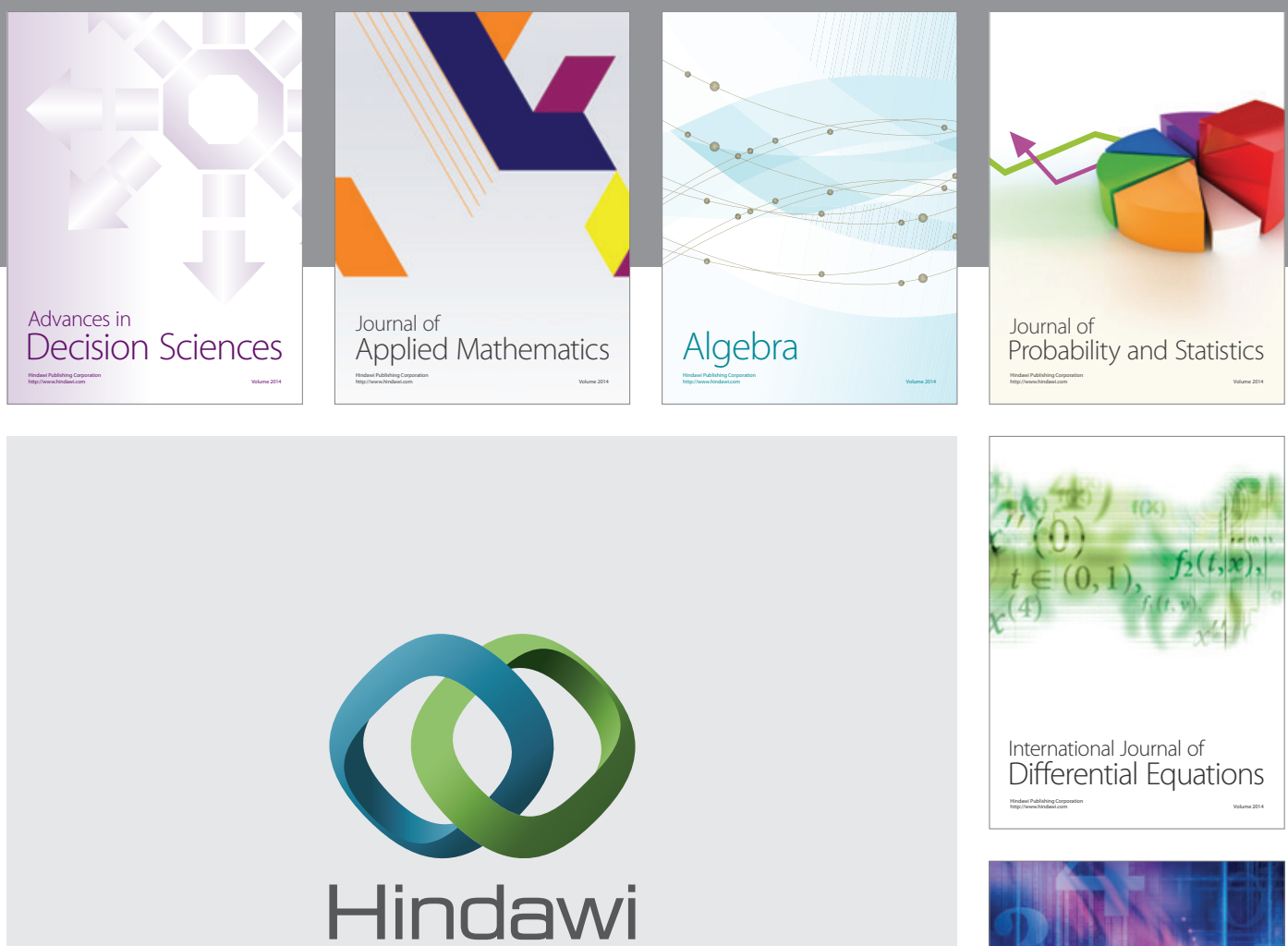

Submit your manuscripts at http://www.hindawi.com
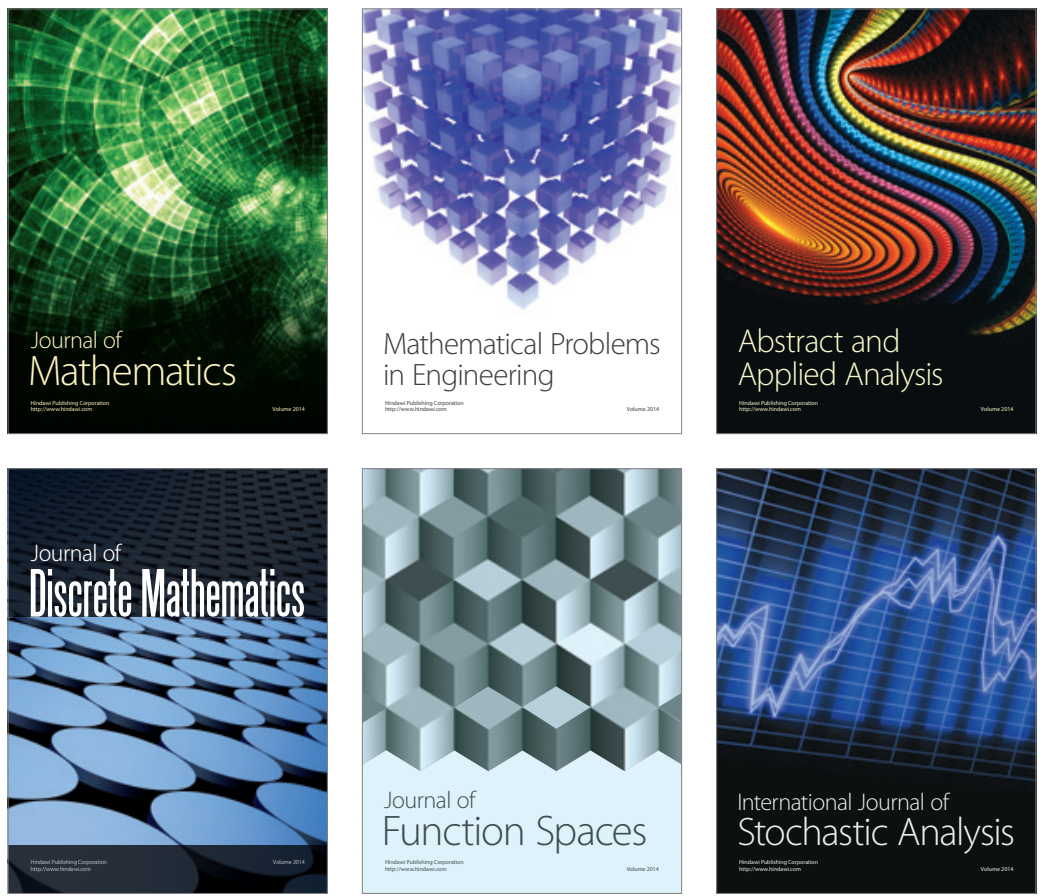

Journal of

Function Spaces

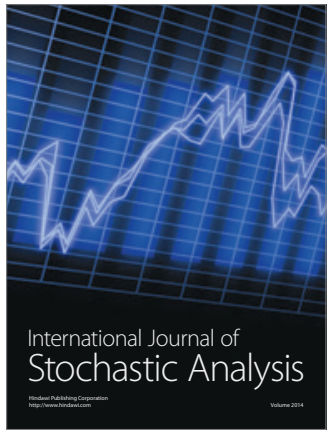

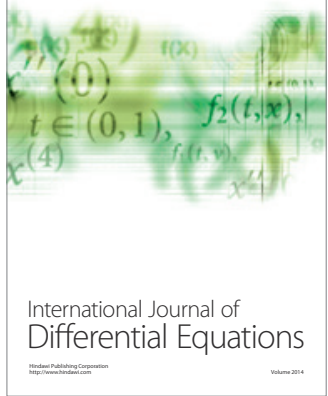
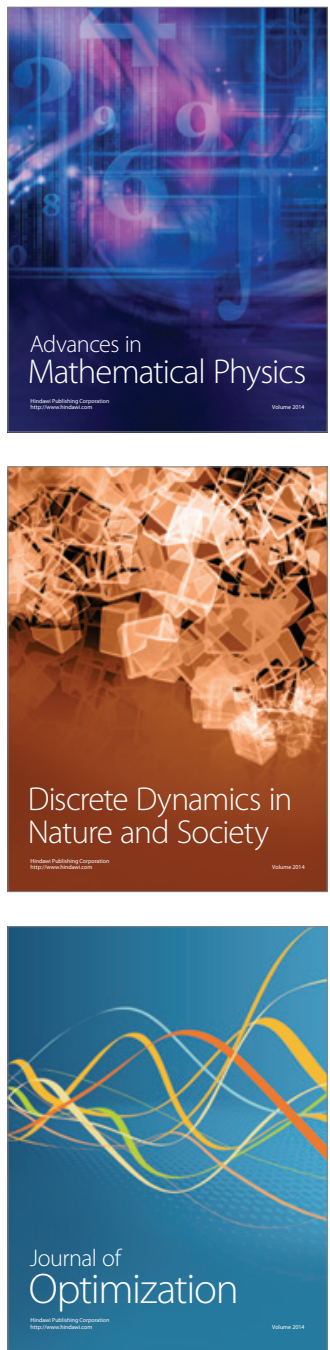